Research Article

\title{
Application of Implantable Polylactic-Co-Glycolic Acid Microcapsule in Repairing Alveolar Bone Defects
}

\author{
Jun Jiang $\left(\mathbb{D},{ }^{1}\right.$ Jianpeng Xiao $\mathbb{D},{ }^{1}$ Dongqing Wang $\mathbb{D},{ }^{2}$ and Huazhong Cai $\mathbb{D}^{1,2,3}$ \\ ${ }^{1}$ School of Pharmacy, Jiangsu University, 301\# Xuefu Road, Zhenjiang 212013, Jiangsu Province, China \\ ${ }^{2}$ Affiliated Hospital of Jiangsu University, Department of Medical Imaging, Zhenjiang 212001, Jiangsu Province, China \\ ${ }^{3}$ Affiliated Hospital of Jiangsu University, Department of Emergency, Zhenjiang 212001, Jiangsu Province, China
}

Correspondence should be addressed to Jun Jiang; jiangjuntcm2007@hotmail.com

Received 19 February 2021; Revised 1 June 2021; Accepted 16 July 2021; Published 28 July 2021

Academic Editor: Jin-Yi Wan

Copyright (C) 2021 Jun Jiang et al. This is an open access article distributed under the Creative Commons Attribution License, which permits unrestricted use, distribution, and reproduction in any medium, provided the original work is properly cited.

\begin{abstract}
Alveolar bone defects (ABDs) were a perennial problem, especially in the aged. Bisphosphonates, especially etidronate sodium (ET), were frequently used in clinical treatment of ABD. However, the oral administration of ET had poor absorption $(<1 \%)$. Therefore, optimization of a suitable dosage form substituted with ET to locally repair the ABD was a straightforward approach. Polylactide-co-glycolide (PLGA) is a biodegradable material and had been used in locally implanted medical devices. Therefore, an ET-PLGA microcapsule may help local delivery and prolong the activity of healing ABD. In this paper, a preparation method of ET-PLGA microcapsule was optimized by the single-factor investigation and response surface method. Subsequently, the rat ABD model was used to evaluate the enhancement effect of these microcapsules. Finally, the optimum parameters were determined as follows: 40\% dichloromethane, $160 \mathrm{mg} / \mathrm{mL}$ PLGA, 10\% internal aqua/oil phase, 4\% PVA, and emulsifying for $10 \mathrm{~min}$. These microcapsules were spherical in shape and fairly monodisperse in a particle size of $27,51 \mu \mathrm{m}(\mathrm{PDI}=0.3)$, encapsulation rate $96.6 \%$, and drug loading 4.58\%. Compared with the ET groups, the total healing volume of ABD in ET-PLGA groups was significantly increased $(P<0.05)$. ET-PLGA microcapsules significantly enhanced the effect of ET on ABD. This study provided important technical support for the treatment of $\mathrm{ABD}$ with bisphosphonates by local administration. This paper has an exploratory significance for the development of water-soluble bioactive components with low bioavailability for ABD.
\end{abstract}

\section{Introduction}

Alveolar bone defects (ABDs) continue to be a perennial problem, especially in the aged [1]. ABD is mainly caused by trauma, infection, periodontal disease, or congenital alveolar fenestration, which affected the mastication ability and quality of life $[2,3]$. Bisphosphonates, antibiotics, or antiinflammatory compounds have been wildly used to cure the $\mathrm{ABD}$, but their ability to effectively achieve alveolar regeneration remains elusive $[4,5]$, especially the bisphosphonates.

In clinical treatment, bisphosphonates are the most commonly prescribed for antiresorptive drugs $[6,7]$; for example, etidronate sodium (ET) was frequently chosen to increase bone mineral density and reduce the risk of fracture and was well tolerated $[8,9]$ by oral administration.
However, its excretion levels by the renal system reached $38 \%$ to $73 \%$ in $24 \mathrm{~h}$ [10]. Furthermore, the poor absorption from the gastrointestinal tract was its major disadvantage, generally less than 1\% [11]. Therefore, optimization of synthetic bone substitutes with ET to locally adjust the imbalance in bone remodeling seemed a straightforward approach to aid bone regeneration in $\mathrm{ABD}$.

Among the available synthetic bone substitutes, polylactide-co-glycolide (PLGA) is an FDA-approved biodegradable material and had been used in locally implanted medical devices, including scaffolds [12-15]. PLGA composites could maintain the structural integrity of in situ placement, provide micro-/macropore space, and stabilize the bioadhesion of clots and quick biodegradation for rapid clearance [16]. PLGA microcapsule is an effective way to locally control drug release and can be easily adapted to 
complex defects in a less-invasive manner compared to conventional surgery [17]. Compared to the conventional drug delivery vector, PLGA also had been extensively applied to other delivery systems such as nanocrystals and microspheres [18-20].

According to the internal structure, PLGA microparticles are classified into microspheres and microcapsules [21]. In microcapsules, vesicular particles consist of a polymer shell surrounding a single core (mononuclear) or multicores which mainly appropriate for encapsulating hydrophilic drugs. PLGA implants gradually released encapsulated drug in situ as they were degradated and prolonged the drug bioactivity through control degradation time [22, 23]. Theoretically, PLGA should be able to encapsulate watersoluble ET and increase its bioavailability and tissue repair by controlling release.

$\mathrm{ABD}$ repair generally lasts several months, so it is necessary to prolong the drug efficacy in the treatment of this disease [24]. An ET-loaded PLGA may help local delivery and prolong the activity of $\mathrm{ABD}$ healing in rats for a long period. However, there have been few reports on the fabrication of this delivery system. In this paper, a preparation method of Water ${ }_{1}\left(\mathrm{~W}_{1}\right) / \mathrm{O}(\mathrm{Oil}) / \mathrm{W}_{2}$ ET-PLGA microcapsule was optimized by the single-factor investigation and response surface method. Subsequently, the rats' ABD model was used to evaluate the enhancement effect of these microcapsules.

\section{Materials and Methods}

2.1. Ethics Statement. All animal experiments strictly comply with the Guidelines for Animal Experimentation of Jiangsu University (Zhenjiang, China), and the protocol was approved by the Animal Ethics Committee of this institution.

2.2. Materials and Chemicals. Etidronate sodium (purity $>99.8 \%$ ) was purchased from Jizhi Biochemical Technology Co., Ltd. (Shanghai, China). PLGA (75/25) was purchased from Jinan Daigang Bioengineering Co., Ltd. (Jinan, Shanghai, China). The chromatographic distilled water was made in our laboratory. Chloral hydrate, polyvinyl alcohol (PVA), dichloromethane (DCM), ethyl acetate (EAC), and $\mathrm{KOH}$ were purchased from Titan Technology Co., Ltd. (Shanghai, China).

2.3. Preparation of ET-PLGA Microcapsules. The ET-PLGA microcapsules were prepared by improved double emulsion solvent-an evaporation method to form $\mathrm{W}_{1} / \mathrm{O} / \mathrm{W}_{2}$ complex emulsion. The customized preparation parameters were set as follows: 40\% DCM (DCM: EAC), $160 \mathrm{mg} / \mathrm{mL}$ PLGA, $10 \%$ internal aqua/oil phase, 4\% PVA, and emulsifying for $10 \mathrm{~min}$. The operation flow is shown in Figure 1.

2.4. Quality Evaluation of Microcapsules. The encapsulation efficiency (EE) of microcapsules was chosen as the main index to evaluate microcapsules, and the drug loading yield (DL) and particle size distribution (PSD) were also considered. EE $(\%)=[$ (amount of drug used in the formulation-Amount of residue in the supernatant)/Amount of drug used in the formulation] $\times 100 \%$. Actual drug loading $=($ Total amount of drugs in microcapsules/Microcapsule weight) $\times 100 \%$.

2.5. Determination of Drug Content. We accurately weighed etidronate sodium, prepared $5 \mathrm{mg} / \mathrm{mL}$ standard solution, diluted to $10 \mu \mathrm{g} / \mathrm{mL}, 40 \mu \mathrm{g} / \mathrm{mL}, 80 \mu \mathrm{g} / \mathrm{mL}, \quad 160 \mu \mathrm{g} / \mathrm{mL}$, $320 \mu \mathrm{g} / \mathrm{mL}$, and $400 \mu \mathrm{g} / \mathrm{mL}$ standard solution. An Ics 600 ion chromatograph (Thermo Fisher Scientific Co., Ltd., Shanghai, China) was used to detect the peak area as the ordinate and the concentration as the abscissa, and linear regression was used to obtain the standard curve equation. In order to determine the residual amount of ET in the supernatant as described in Figure 1,5 mL of the supernatant of ET-PLGA microcapsule preparation was obtained, successively filtrated by using a $\mathrm{C}_{18}$ SPE column and $0.22 \mu \mathrm{m}$ aqueous membrane, and then, injected into an ion chromatograph for analysis.

2.6. Single-Factor Experiment. The entrapment rate as the main index and the main factors affecting the formation of microcapsules, such as oil phase composition (DCM: EAC), PLGA concentration, volume ratio of internal water phase to oil phase (RWO), emulsification time, and PVA concentration, were investigated. During the single-factor investigation, the encapsulation rate and particle size were measured as evaluation indicators.

2.7. Response Surface Experiment. Based on single-factor experiment, response surface experiment was designed by selecting 3 factors (Proportion of DCM, PLGA concentration and RWO) which have an obvious influence on the quality of microcapsules. The response surface was designed by Box-Behnken, and the encapsulation rate was taken as the index.

2.8. Animals and Administration. Thirty SD rats $(220 \pm 20 \mathrm{~g})$ were supplied by the Laboratory Animal Center of Jiangsu University. Before the experiment, all rats were given 14 days of adaptation period with a standard laboratory rodent diet (calcium content $0.5 \%$ ) and tap water under climate-controlled conditions (55\% humidity, $25^{\circ} \mathrm{C}$, and 12 hours alternating day and night). After the accommodation period, the rats were anesthetized with $10 \%$ urethane $(10 \mathrm{~mL} / \mathrm{kg})$ by intraperitoneal injection. After fixation, the alveolar bone of rat was exposed and a round hole bone defect was made by using a dental drill ( $T_{3}$, Sirona Dental Systems Co., Ltd., Shanghai, China) near the first molar. In order to prevent the high temperature of the operation area, the rats' ABD was made by intermittent and low-speed drilling. After establishing the ABD model, the ET powder or prepared ETPLGA microcapsule were implanted into the bone defect immediately, and the gingiva was sutured carefully. Rats were divided into 6 groups $(n=5)$; they were the blank control group (CON), ABD model group (ABD), ET low- 


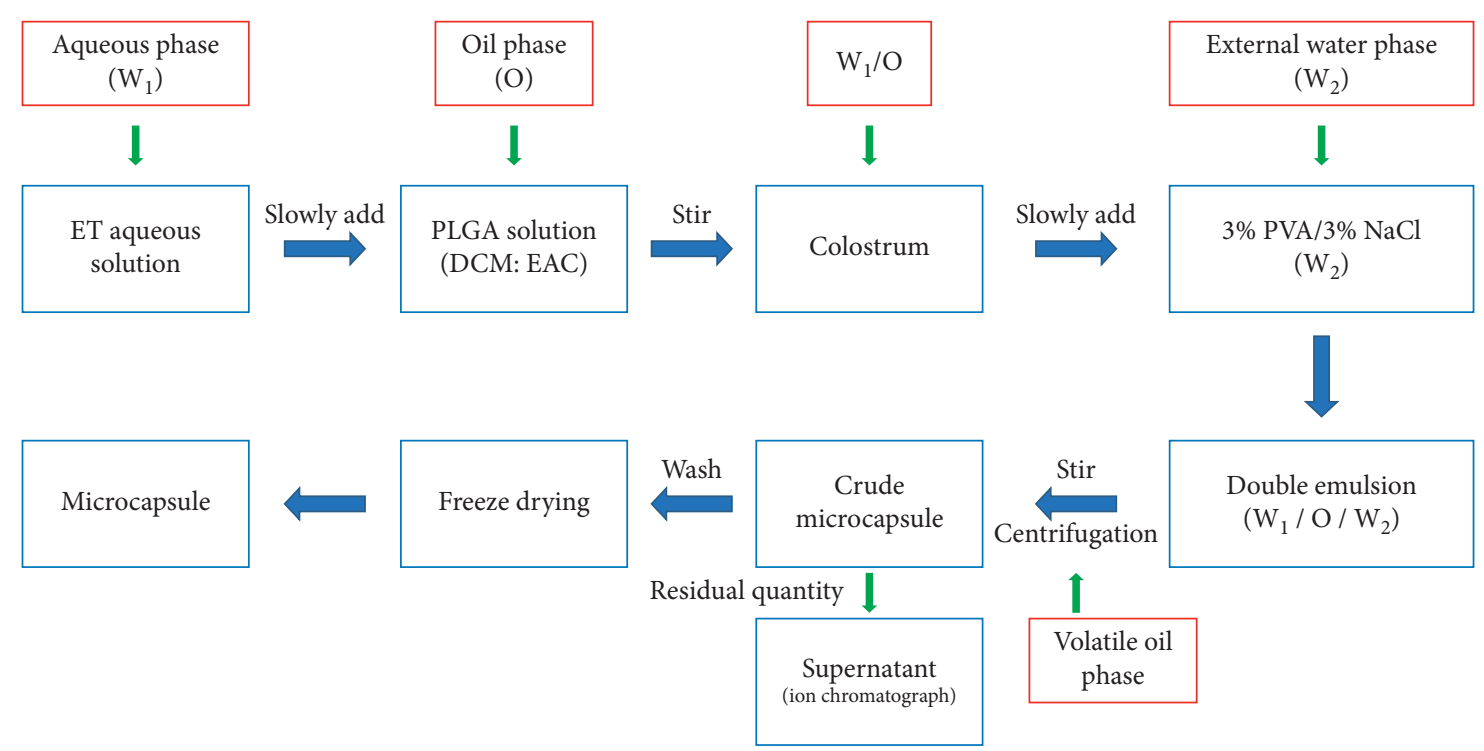

Figure 1: Preparation flow chart of ET-PLGA microcapsules.

dose group (ET-L, $2 \mathrm{mg}$ ), ET high-dose group (ET-H, $10 \mathrm{mg}$ ), ET-PLGA low-dose group (ET-PLGA-L, $2 \mathrm{mg}$ ), and ET-PLGA high-dose group (ET-PLGA-L-H, $10 \mathrm{mg}$ ). In the CON group, no diaphragm was implanted and the wound was sutured directly. All the experimental protocols were approved by the Animal Ethics Committee of Jiangsu University.

2.9. Collection and Analysis of Alveolar Bone Samples. Within one month after the operation, cone-beam computed tomography (CB-CT, kawa i-cat 17-19, Imaging science international LLC, USA) was used to scan the ABD of rats every week. The main parameters of CB-CT were as follows: reconstruction solvent size was $8 \mathrm{~cm} \times 8 \mathrm{~cm}$ (diameter $\times$ height), resolution was 125 pixels, exposure was $\mathrm{mAs}=37.07, \mathrm{KVP}=120$, and acquisition time $=26.9 \mathrm{sec}-$ onds. Mimics Research 20.0 software was applied to analyze the CT image and calculate defect volume.

2.10. Statistical Analysis of Data. Statistical analyses were conducted by one-way ANOVA followed by Tukey's test (GraphPad Prism 5.0) for comparing all groups. Data were presented as mean value \pm SD. The $P$ value $<0.05$ was considered as statistically significant.

\section{Results}

3.1. Methodological Validation. Ion chromatography was established for the determination of ET residue in the supernatant by an external standard method (Figure 2). The mobile phase was $30 \mathrm{mmol} / \mathrm{L} \mathrm{KOH}$ solution with isocratic elution. The regression equation was obtained by linear regression of concentration $Y$ with peak area $X$. The standard curve was $Y=0.251, X-0.184, R^{2}=0.9996$. The results showed that there is a good linear relationship between $10 \mu \mathrm{g} / \mathrm{mL}$ and $400 \mu \mathrm{g} / \mathrm{mL}$. The 3 or 10 times of the relative standard deviation of the analytical blank values was calculated as the limit of detection
(LOD) and limit of quantitation (LOQ). The LOD and LOQ were $0.6 \mathrm{ng}$ and $2.1 \mathrm{ng}$, respectively. One sampling solution was treated according to Section 2.5 and then injected into an ion chromatograph for 6 times continuously to record the peak area of ET. The RSD was $2.34 \%$ which indicated that the precision is fine (Table S1). Similarly, when the sample solution was injected at $0,2,4,8,16$, and $24 \mathrm{~h}$, the RSD of the peak area of ET was $0.27 \%$, indicating that ET was stable within $24 \mathrm{~h}$ under room temperature (Table S2). For repeatability, six samples with the same concentration were determined and the RSD of their contents was $0.37 \%$ (Table S3), indicating a good repeatability. The sample with known concentration was chosen, and, respectively, added into ET according to $1: 1,1: 2$, and $1: 4$ times, and the recovery rate was calculated ([Measured quantity-Original quantity] $\times 100 /$ Amount added, \%). The recovery was $99.6 \%$ with the RSD of $1.81 \%$ (Table S4). All these results showed that the ion chromatography method established in this paper can be well applied to optimize the preparation of ET-PLGA microcapsules.

3.2. Single-Factor Results. The oil phases $(30 \%, 45 \%, 60 \%$, and $75 \%$ dichloromethane, Table 1), PLGA concentrations $(80,120,160$, and $200 \mathrm{mg} / \mathrm{mL}$, Table 2$)$, PVA concentrations $(1 \%, 2 \%, 3 \%$, and $4 \%$, Table 3$)$, emulsification times $(5,10$, 15 , and $20 \mathrm{~min}$, Table 4), and RWO (v/v 5\%, 10\%, 15\%, and $20 \%$, Table 5) were investigated by EE (\%) and particle size $(\mathrm{nm})$. The single factor results indicated that the best encapsulation efficiency was obtained when the proportion of DCM, PLGA, PVA, emulsification time, and RWO was $30 \%$, $200 \mathrm{mg} / \mathrm{mL}, 4 \%, 10 \mathrm{~min}$, and 5\%, respectively.

3.3. Response Surface Test Results. The proportion of DCM and the concentration of PLGA and RWO were the three factors that had an obvious influence on the quality of microcapsules. The Box-Behnken software was used to design response surface experiment (Table 6), and the encapsulation 


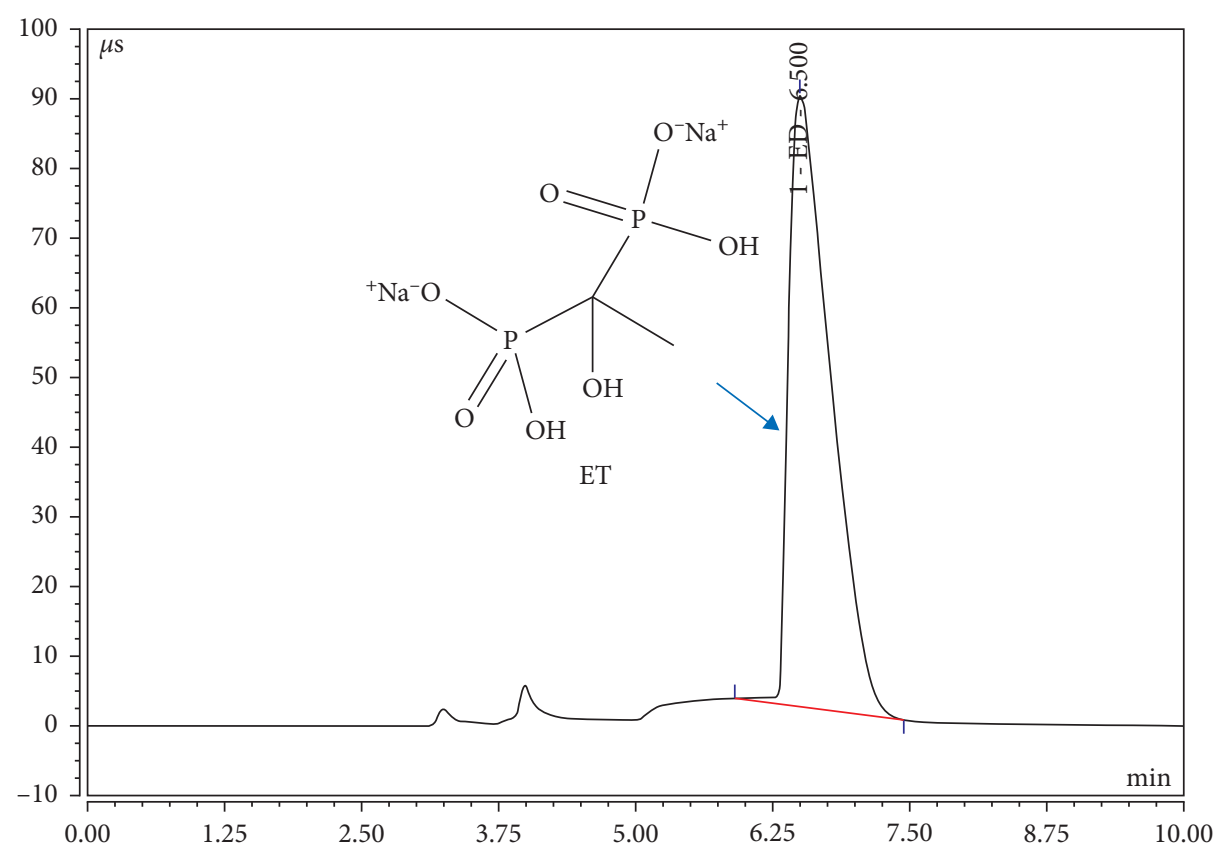

(a)

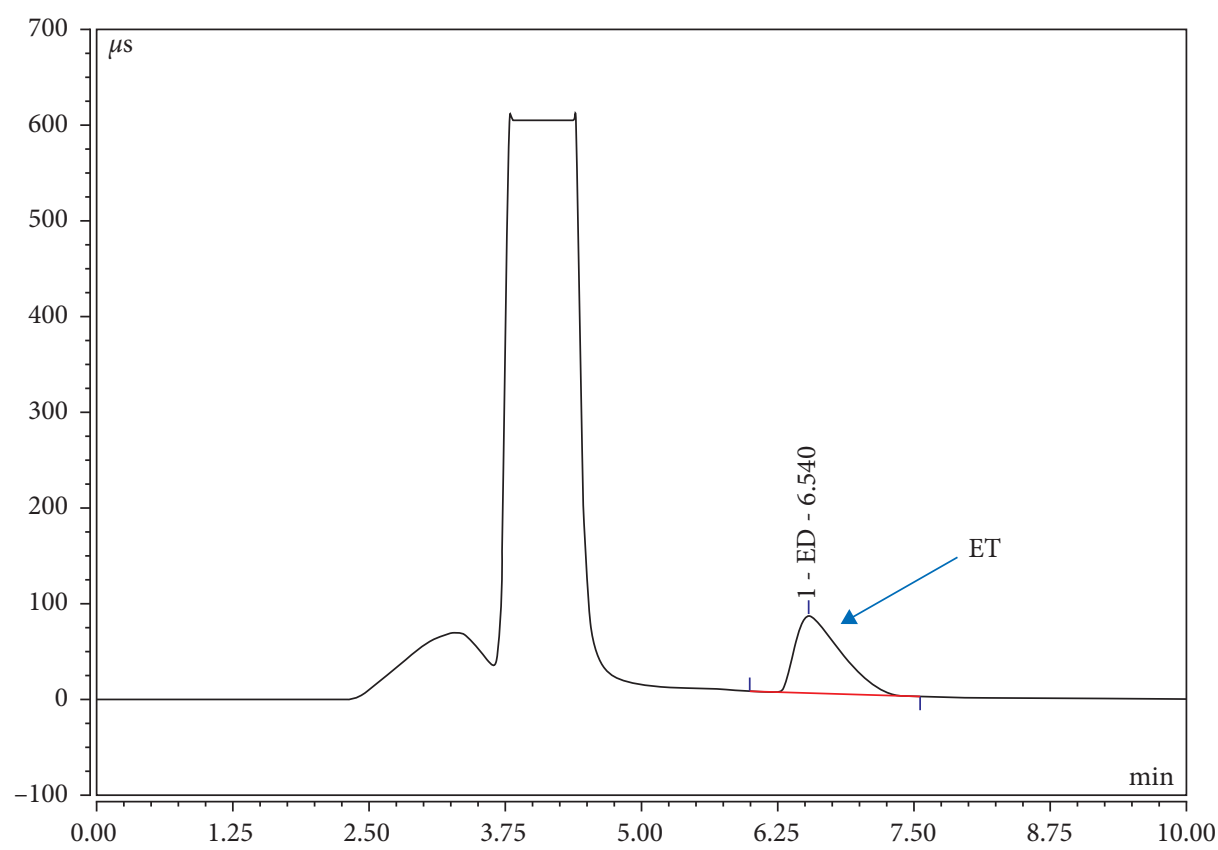

(b)

FIgURE 2: Determination of sodium etidronate by ion chromatography. (a) Chromatogram of the ET standard solution; (b) chromatogram of ET detection in samples.

rate was taken as the evaluation index. The predicted results of response surface showed that when the oil phase composition (DCM: EAC) was 40\%, PLGA was $160 \mathrm{mg} / \mathrm{mL}$ and RWO was $10 \%$, and the EE\% was up to $97.83 \%$ (Figure 3 ).

3.4. Validation of Optimum Preparation. According to the results of single-factor and response surface experiments, the optimum parameters were determined as follows: $40 \%$
DCM, $160 \mathrm{mg} / \mathrm{mL}$ PLGA, 10\% RWO, 4\% PVA, and emulsifying time $10 \mathrm{~min}$. Based on the optimized results, 3 batches of ET-PLGA microcapsules were prepared in parallel, and their EE, DL, particle size, and morphology were detected in turn. The results demonstrated that the average encapsulation rate was $96.6 \%$ (RSD, $0.46 \%$ ) and the average $\mathrm{DL}$ was $4.58 \%$ (RSD, $0.98 \%$ ). Under the microscope and scanning electron microscope, the surface of ET-PLGA microcapsules was smooth without adhesion (Figure 4 ). The 
TABLE 1: The effect of oil phase composition on the preparation of PLGA microcapsules.

\begin{tabular}{lcc}
\hline Proportion of DCM (\%) & EE (\%) & Particle size (nm) \\
\hline 30 & 87.11 & 12085.61 \\
45 & 84.94 & 21872.26 \\
60 & 84.59 & 27462.43 \\
75 & 80.92 & 27174.97 \\
\hline
\end{tabular}

TABle 2: Effect of PLGA concentration on the preparation of PLGA microcapsules.

\begin{tabular}{lcc}
\hline PLGA concentration $(\mathrm{mg} / \mathrm{mL})$ & EE $(\%)$ & Particle size $(\mathrm{nm})$ \\
\hline 80 & 74.94 & 7784.28 \\
120 & 87.55 & 9500.88 \\
160 & 84.74 & 25136.59 \\
200 & 92.94 & 25732.73 \\
\hline
\end{tabular}

TABLE 3: Effect of PVA concentration on the preparation of PLGA microcapsules.

\begin{tabular}{lcr}
\hline PVA concentration (\%) & EE (\%) & Particle size (nm) \\
\hline 1 & 90.88 & 56577.15 \\
2 & 89.67 & 95270.58 \\
3 & 82.65 & 19958.97 \\
4 & 98.79 & 11593.56 \\
\hline
\end{tabular}

TABLE 4: Effect of emulsification time on the preparation of PLGA microcapsules.

\begin{tabular}{lcc}
\hline Emulsification time (min) & EE (\%) & Particle size (nm) \\
\hline 5 & 83.53 & 18711.51 \\
10 & 86.88 & 82029.78 \\
15 & 84.94 & 13068.48 \\
20 & 84.94 & 18896.24 \\
\hline
\end{tabular}

TABLE 5: Effect of volume ratio of the internal water phase to oil phase on the preparation of PLGA microcapsules.

\begin{tabular}{lcc}
\hline $\begin{array}{l}\text { Proportion of the internal water phase } \\
(\%)\end{array}$ & EE (\%) & $\begin{array}{c}\text { Particle size } \\
(\mathrm{nm})\end{array}$ \\
\hline 5 & 93.47 & 38459.89 \\
10 & 78.37 & 19318.54 \\
15 & 85.03 & 20178.52 \\
20 & 78.23 & 20780.43 \\
\hline
\end{tabular}

particle size was $27.51 \mu \mathrm{m}$ (RSD, $1.2 \%$ ), and the polydispersity index (PDI) was below 0.3 , indicating that the distribution of the particles was narrow. After measuring zeta potential, it was found that the average potential of the particle was below $-38 \mathrm{mV}$, indicating that its property was relatively stable (Table 7 ).

3.5. Healing Effect on $A B D$. After administration, the healing volume was calculated once a week for 4 consecutive times. When measuring the healing volume (HV) of bone defect weekly, the calculation method was to subtract the volume of defect (VD) in the previous week from the VD in the current
TABLE 6: Response surface experiment design.

\begin{tabular}{lccccc}
\hline Std & Run & A & B & C & EE\% \\
\hline 2 & 1 & 1 & -1 & 0 & 96.88 \\
7 & 2 & -1 & 0 & 1 & 93.49 \\
1 & 3 & -1 & -1 & 0 & 94.90 \\
16 & 4 & 0 & 0 & 0 & 95.78 \\
15 & 5 & 0 & 0 & 0 & 96.88 \\
10 & 6 & 0 & 1 & -1 & 96.78 \\
6 & 7 & 1 & 0 & -1 & 96.88 \\
4 & 8 & 1 & 1 & 0 & 96.78 \\
11 & 9 & 0 & -1 & 1 & 96.94 \\
5 & 10 & -1 & 0 & -1 & 96.95 \\
3 & 11 & -1 & 1 & 0 & 96.93 \\
14 & 12 & 0 & 0 & 0 & 95.28 \\
9 & 13 & 0 & -1 & -1 & 96.97 \\
17 & 14 & 0 & 0 & 0 & 96.92 \\
8 & 15 & 1 & 0 & 1 & 96.97 \\
13 & 16 & 0 & 0 & 0 & 96.91 \\
12 & 17 & 0 & 1 & 1 & 96.94 \\
\hline
\end{tabular}

"-1": $20 \%$ DCM, PLGA $160 \mathrm{mg} / \mathrm{mL}, 5 \%$ proportion of the internal water phase; "- -0 ": $30 \%$ DCM, PLGA $180 \mathrm{mg} / \mathrm{mL}, 7.5 \%$ proportion of the internal water phase; "1": $40 \%$ DCM, PLGA $200 \mathrm{mg} / \mathrm{mL}, 10 \%$ proportion of the internal water phase. 


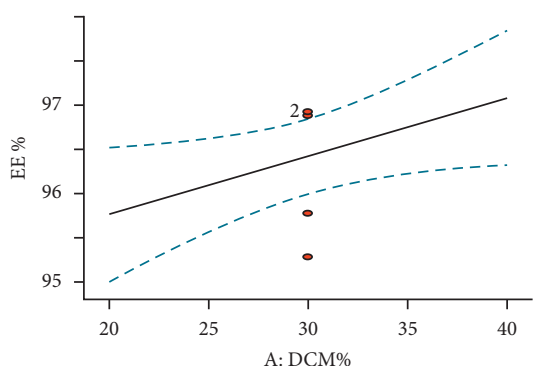

(a)

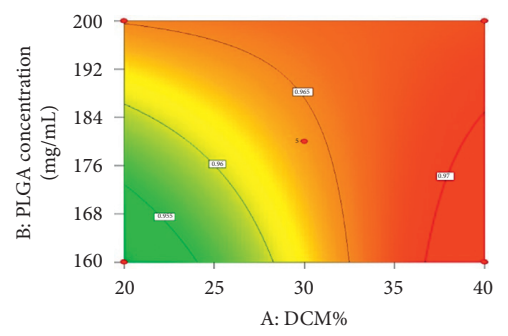

(d)

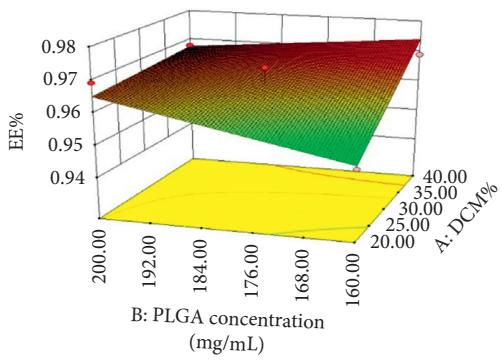

(g)

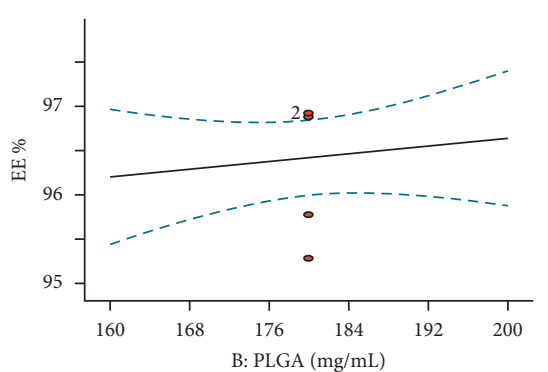

(b)

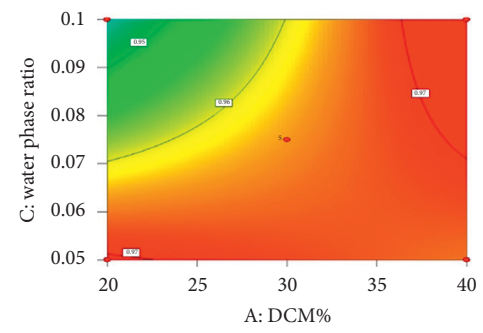

(e)

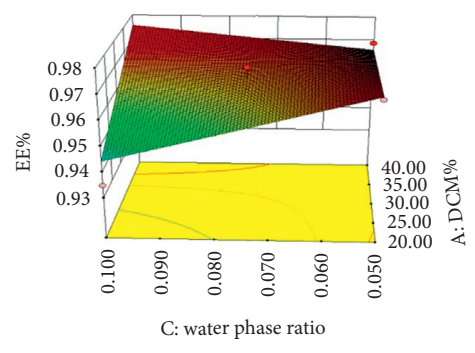

(h)

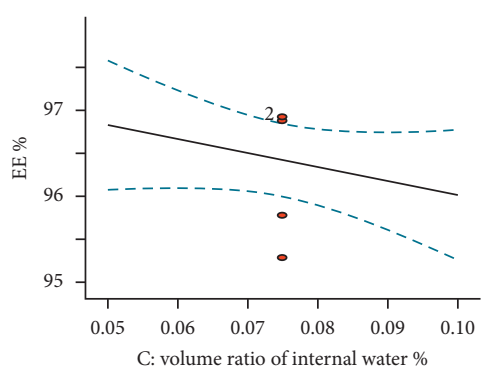

(c)

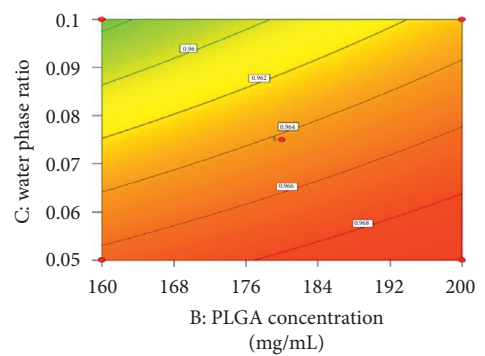

(f)

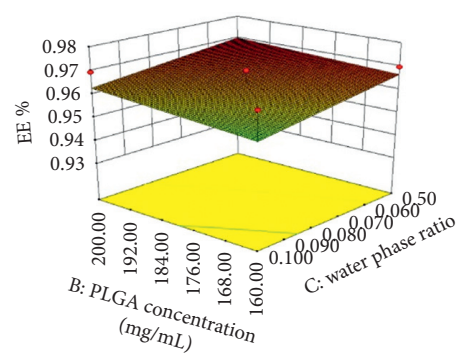

(i)

FIGURE 3: Response surface optimization results. (a) The effect of DCM ratio on EE\%; (b) the effect of PLGA concentration on EE\%; (c) the influence of the ratio of internal water phase to EE\%; (d) 2D panel of PLGA-DCM; (e) 2D panel of internal water phase-DCM; (f) 2D panel of internal water phase-PLGA; and (g)-(i) 3D response surface plots.

week, such as $\mathrm{HV}_{n}=\mathrm{VD}_{n-1}-\mathrm{VD}_{n}, n=1,2,3,4$. The total healing volume (THV) was calculated as $\mathrm{THV}=\mathrm{HV}_{1}+\mathrm{HV}_{2}+\mathrm{HV}_{3}+\mathrm{HV}_{4}$. The effects of ET and ETPLGA microcapsules on the healing of $\mathrm{ABD}$ were compared by total healing volume (Figure 5). Compared with the ABD group $\left(0.478 \pm 0.100 \mathrm{~mm}^{3}\right)$, the total healing volume in all treatment groups was significantly increased $(P<0.01)$. Compared with the ET-L group $\left(0.828 \pm 0.075 \mathrm{~mm}^{3}\right)$, the total healing volume of ET-PLGA-L $\left(1.133 \pm 0.175 \mathrm{~mm}^{3}\right)$ was enhanced significantly $(P<0.05)$. Compared with the ET-H group $\left(1.223 \pm 0.083 \mathrm{~mm}^{3}\right)$, the total healing volume of ETPLGA-H $\left(1.528 \pm 0.113 \mathrm{~mm}^{3}\right)$ was also significantly improved $(P<0.05)$. Additionally, there was no abnormal infection, ulceration, or death in the ET-PLGA microcapsule group during the whole experiment.

\section{Discussion}

Compared with the traditional PLGA microspheres, the preparation of PLGA microcapsules with water-phase nuclei has higher technical difficulties [25]. PLGA microspheres have shortcomings in drug loading, encapsulation efficiency, and drug release, especially for water-soluble drugs and hydrophilic macromolecules [26-28]. In this paper, ETPLGA microcapsules were prepared by double emulsion-solvent evaporation $\left(\mathrm{W}_{1} / \mathrm{O} / \mathrm{W}_{2}\right)$. PLGA polymers were dissolved in a mixture of DCM and EAC (volatile), and then, ET aqueous solution was slowly dripped into it to form an internal aqueous phase, thus forming a $\mathrm{W}_{1} / \mathrm{O}$ colostrum. Then, the $\mathrm{W}_{1} / \mathrm{O}$ colostrum was slowly dripped into the aqueous solution containing 3\% PVA and $3 \% \mathrm{NaCl}$ to form 

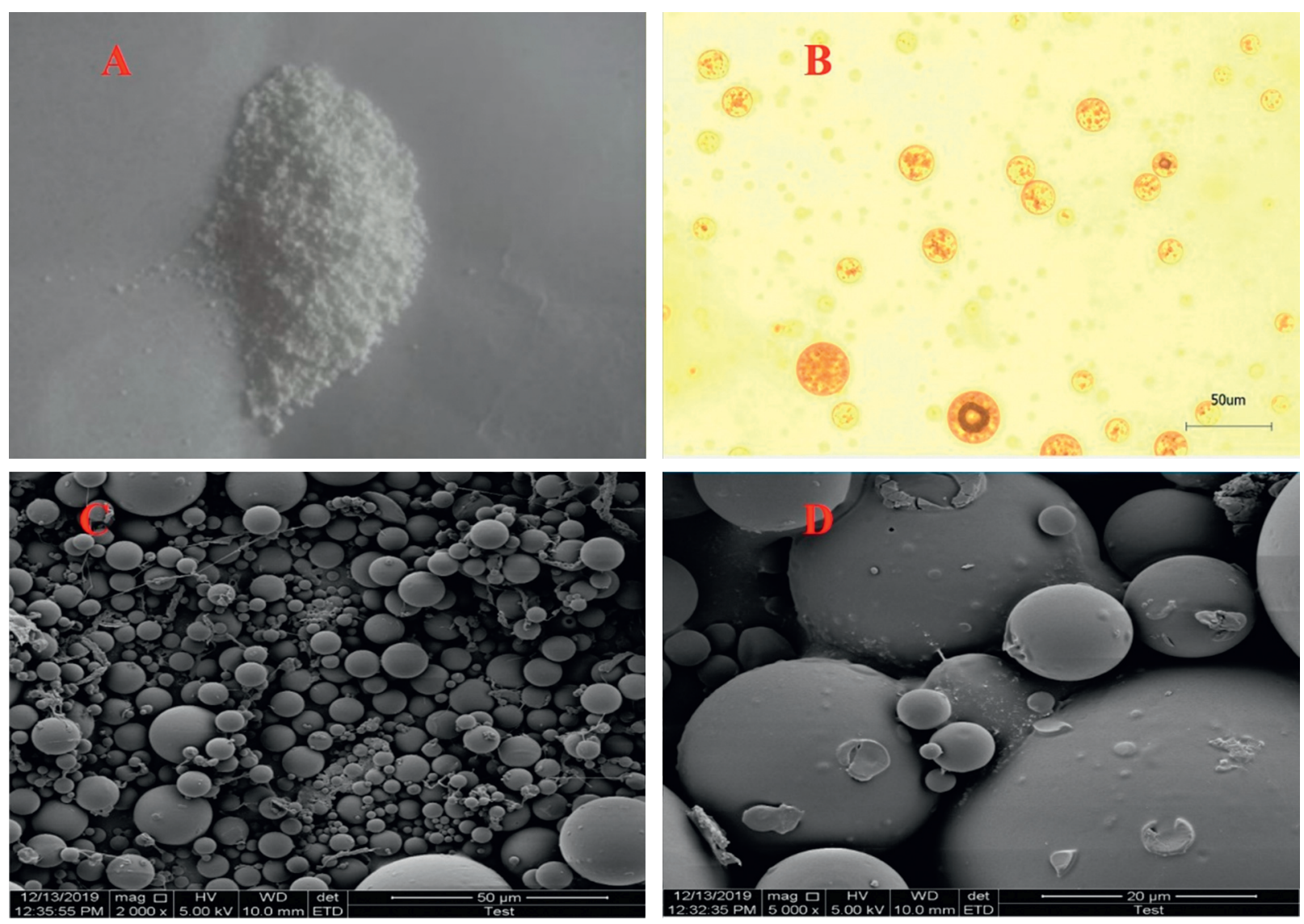

Figure 4: The morphology of ET-PLGA microcapsules. (a) Freeze-dried powder of ET-PLGA microcapsules; (b) the morphology of ET-PLGA microcapsules under an upright microscope; and (c) the morphology of ET-PLGA microcapsules under a scanning electron microscope.

TABLe 7: Particle size and potential of ET-PLGA microcapsules.

\begin{tabular}{|c|c|c|c|c|c|}
\hline Batch & Particle size $(\mathrm{nm})$ & Polydispersity & Zeta potential $(\mathrm{mV})$ & $\mathrm{EE} \%$ & DL\% \\
\hline 1 & 2740 & 0.26 & -38.29 & 96.65 & 4.64 \\
\hline 2 & 2717 & 0.30 & -38.63 & 97.12 & 4.57 \\
\hline 3 & 2795 & 0.29 & -38.33 & 96.03 & 4.53 \\
\hline Average value & 2751 & 0.28 & -38.42 & 96.60 & 4.58 \\
\hline
\end{tabular}

the $\mathrm{W}_{1} / \mathrm{O} / \mathrm{W}_{2}$ composite emulsion. Finally, upon DCM and EAC evaporation from the internal oil phase droplets, the solubility of PLGA polymers decreased gradually leading to phase separation and migration to the interface surrounding water droplets.

It was found that the amount of PLGA had a significant impact on the particle size of microcapsules [29]. With the increase of PLGA concentration, the size of microcapsules decreased from several microns to several hundred nanometers. PLGA not only acted as a drug carrier but also played the role of an emulsifier when the internal water phase was encapsulated into the oil phase [30-32]. It could be inferred that the stability of the colostrum was one of the key factors affecting the EE of microcapsules. In addition, due to the presence of PVA in the external water phase, it had the function of a surfactant. When the colostrum was added into the external aqueous phase to prepare the compound emulsion, the outer wall of this prepared microcapsule was compact and uniform [33-35].

Determination of ET and calculation of bone defect volume were the other two technical difficulties in this paper. Fortunately, an ion chromatography method was successfully established, which completed the analysis of a single sample within $10 \mathrm{~min}$ with high precision. This powerful analysis method provided important technical support for the optimization of microcapsules. On the other hand, evaluation of the enhancement effect of microcapsules on $\mathrm{ABD}$ repair lasted for 1 months and the scanning by CB-CT was needed every week. CB-CT, as a modern noninvasive imaging technique, was widely used in radiology, orthopedics, dentistry, and image-guided radiation therapy $[36,37]$. Compared with micro-CT and high-resolution CT, 

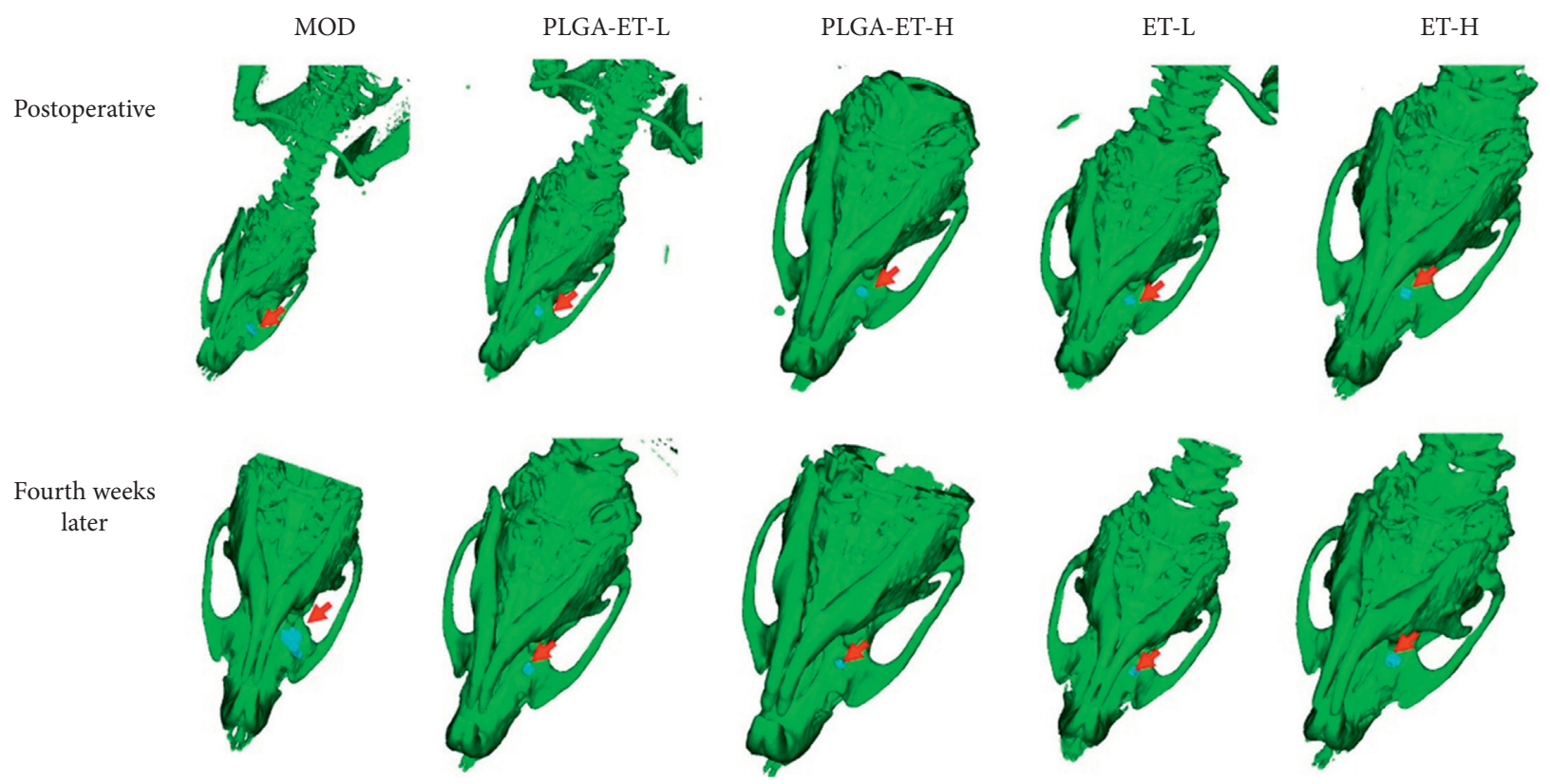

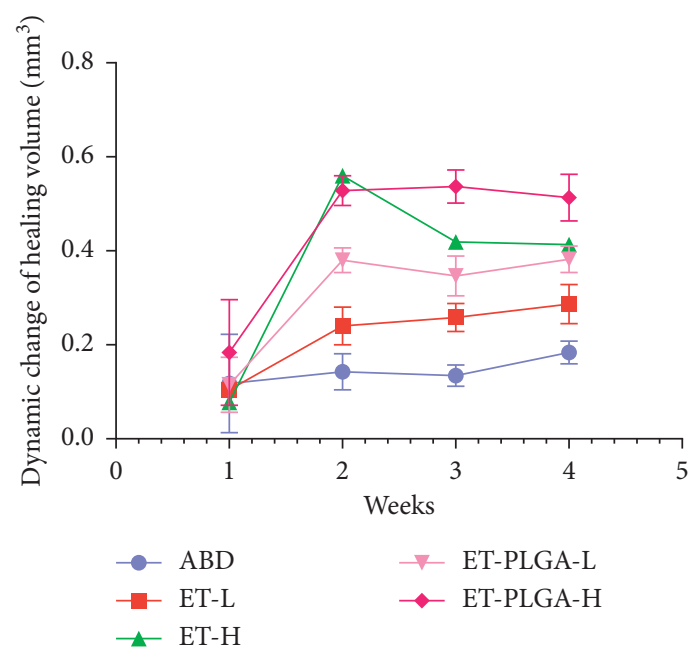

(b)

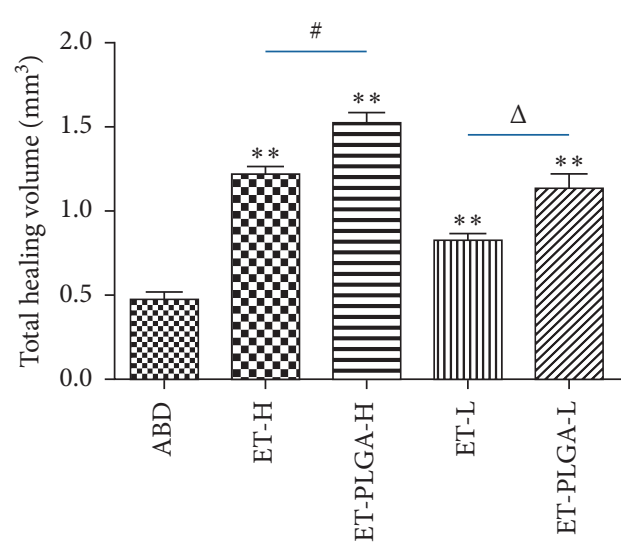

(c)

Figure 5: The effect of ET-PLGA microcapsules on alveolar bone defects by CB-CT $(n=5)$. (a) CB-CT images of ABD in rats at different time periods; (b) dynamic changes of healing volume $\left(\mathrm{mm}^{3}\right)$ of ET-PLGA microcapsules in ABD rats; and (c) the total healing volume $\left(\mathrm{mm}^{3}\right)$ of ABD in each group. ${ }^{* *}$, compared with the ABD model group, $P<0.01$; \#, compared with the ET-H group, $P<0.05$; and $\Delta$, compared with the ET-L group, $P<0.05$.

CB-CT recovered imaging parameters accurately, leading to superior image quality [38]. CB-CT provided strong technical support for the evaluation of synergism of ET-PLGA microcapsules in this study.

\section{Conclusions}

An improved double emulsion/solvent evaporation approach was employed to prepare ET-PLGA microcapsules successfully. These microcapsules were spherical in shape and fairly monodisperse in size with a mean particle size of $27.51 \mu \mathrm{m} \quad(\mathrm{PDI}=0.3)$. The results demonstrated that the average encapsulation rate was $96.6 \%$ and the average DL was $4.58 \%$. ET-PLGA microcapsules significantly enhanced the effect of ET on alveolar bone defects. This study provided an important research foundation for the local use of bisphosphonates. More importantly, the implantable PLGA microcapsule system constructed in this study was especially suitable for water-soluble active ingredients (polysaccharides or peptides) with low oral bioavailability in natural medicine.

\section{Data Availability}

The data used to support the findings of this study are available from the corresponding author upon request. 


\section{Conflicts of Interest}

All authors had no conflicts of interest regarding the publication of this study.

\section{Authors' Contributions}

Jun Jiang and Jianpeng Xiao contributed equally to this paper.

\section{Acknowledgments}

This work was supported by the National Natural Science Foundation of China (No. 81703773) and Natural Science Foundation of Jiangsu Province (No. BK20170560).

\section{Supplementary Materials}

Table S1. The precision of ET by ion chromatography. Table S2. The stability of ET by ion chromatography. Table S3. The repeatability of ET by ion chromatography. Table S4. The Recovery test of ET by ion chromatography. (Supplementary Materials)

\section{References}

[1] L. Wang, W. Xu, Y. Chen, and J. Wang, "Alveolar bone repair of rhesus monkeys by using BMP-2 gene and mesenchymal stem cells loaded three-dimensional printed bioglass scaffold," Scientific Reports, vol. 9, no. 1, Article ID 18175, 2019.

[2] H. H. Tüz, O. Koç, S. E. Meral, and A. S. El, "Reconstruction and implant-supported rehabilitation of an iatrogenically caused maxillary alveolar defect," Implant Dentistry, vol. 28, no. 5, pp. 510-513, 2019.

[3] J. Pan, J. Deng, Y. Luo et al., "Thermosensitive hydrogel delivery of human periodontal stem cells overexpressing platelet-derived growth factor-BB enhances alveolar bone defect repair," Stem Cells and Development, vol. 28, no. 24, pp. 1620-1631, 2019.

[4] G. Bhattarai, S. B. Poudel, S.-H. Kook, and J.-C. Lee, "Antiinflammatory, anti-osteoclastic, and antioxidant activities of genistein protect against alveolar bone loss and periodontal tissue degradation in a mouse model of periodontitis," Journal of Biomedical Materials Research Part A, vol. 105, no. 9, pp. 2510-2521, 2017.

[5] P. Goes, N. A. Lima, J. A. G. Rodrigues, N. M. B. Benevides, G. A. C. Brito, and V. Lima, "Anti-inflammatory and antiresorptive effects of atorvastatin on alveolar bone loss in wistar rats," Brazilian Dental Journal, vol. 27, no. 3, pp. 267-272, 2016.

[6] L. Liu, W. Tao, W. Pan et al., "Hydroxysafflor yellow A promoted bone mineralization and inhibited bone resorption which reversed glucocorticoids-induced osteoporosis," Biomed Research International, vol. 2018, Article ID 6762146, 2018.

[7] J. Jiang, S. Xiao, X. Xu, H. Ma, C. Feng, and X. Jia, "Isomeric flavonoid aglycones derived from epimedii folium exerted different intensities in anti-osteoporosis through OPG/ RANKL protein targets," International Immunopharmacology, vol. 62, pp. 277-286, 2018.

[8] R. R. Recker and J. Barger-Lux, "Risedronate for prevention and treatment of osteoporosis in postmenopausal women,"
Expert Opinion on Pharmacotherapy, vol. 6, no. 3, pp. 465477, 2005.

[9] M. P. Ettinger, "Aging bone and osteoporosis," Archives of Internal Medicine, vol. 163, no. 18, pp. 2237-2246, 2003.

[10] R. G. G. Russell, N. B. Watts, F. H. Ebetino, and M. J. Rogers, "Mechanisms of action of bisphosphonates: similarities and differences and their potential influence on clinical efficacy," Osteoporosis International, vol. 19, no. 6, pp. 733-759, 2008.

[11] A. Ezra and G. Golomb, "Administration routes and delivery systems of bisphosphonates for the treatment of bone resorption," Advanced Drug Delivery Reviews, vol. 42, no. 3, pp. 175-195, 2000.

[12] F. Danhier, E. Ansorena, J. M. Silva, R. Coco, A. Le Breton, and V. Préat, "PLGA-based nanoparticles: an overview of biomedical applications," Journal of Controlled Release, vol. 161, no. 2, pp. 505-522, 2012.

[13] P. Gentile, V. Chiono, I. Carmagnola, and P. Hatton, "An overview of poly (lactic-co-glycolic) acid (PLGA)-based biomaterials for bone tissue engineering," International Journal of Molecular Sciences, vol. 15, no. 3, pp. 3640-3659, 2014.

[14] H. K. Makadia and S. J. Siegel, "Poly lactic-co-glycolic acid (PLGA) as biodegradable controlled drug delivery carrier," Polymers, vol. 3, no. 3, pp. 1377-1397, 2011.

[15] J. Naghipoor and T. Rabczuk, "A mechanistic model for drug release from PLGA-based drug eluting stent: a computational study," Computers in Biology and Medicine, vol. 90, pp. 15-22, 2017.

[16] J.-C. Park, U. M. E. Wikesjö, K.-T. Koo et al., "Maturation of alveolar bone following implantation of an rhGDF-5/PLGA composite into 1-wall intra-bony defects in dogs: 24-week histometric observations," Journal of Clinical Periodontology, vol. 39, no. 6, pp. 565-573, 2012.

[17] Y. Yao, F. Kauffmann, S. Maekawa et al., "Sclerostin antibody stimulates periodontal regeneration in large alveolar bone defects," Scientific Reports, vol. 10, no. 1, p. 16217, 2020.

[18] L. Chen, L. Mei, D. Feng et al., "Anhydrous reverse micelle lecithin nanoparticles/PLGA composite microspheres for long-term protein delivery with reduced initial burst," Colloids and Surfaces B: Biointerfaces, vol. 163, pp. 146-154, 2018.

[19] Q. Yang, Y. Wu, F. Lan et al., "Hollow superparamagnetic PLGA/ $\mathrm{Fe}_{3} \mathrm{O}_{4}$ composite microspheres for lysozyme adsorption," Nanotechnology, vol. 25, no. 8, Article ID 085702, 2014.

[20] V. M. Gaspar, A. F. Moreira, E. C. Costa et al., "Gas-generating TPGS-PLGA microspheres loaded with nanoparticles (NIMPS) for co-delivery of minicircle DNA and anti-tumoral drugs," Colloids and Surfaces B: Biointerfaces, vol. 134, pp. 287-294, 2015.

[21] M. S. Shive and J. M. Anderson, "Biodegradation and biocompatibility of PLA and PLGA microspheres," Advanced Drug Delivery Reviews, vol. 28, no. 1, pp. 5-24, 1997.

[22] O. A. Sindeeva, O. I. Gusliakova, O. A. Inozemtseva et al., "Effect of a controlled release of epinephrine hydrochloride from PLGA microchamber array: in vivo studies," ACS Applied Materials \& Interfaces, vol. 10, no. 44, pp. 37855-37864, 2018.

[23] M. J. Dorta, A. Oliva, O. Munguía, M. Llabrés, and J. B. Fariña, "In-vitro release of fluoropyrimidines from PLGA film implants," The Journal of Pharmacy and Pharmacology, vol. 54, no. 6, pp. 757-763, 2002.

[24] S. K. Boda, Y. Almoshari, H. Wang et al., "Mineralized nanofiber segments coupled with calcium-binding BMP-2 peptides for alveolar bone regeneration," Acta Biomaterialia, vol. 85, pp. 282-293, 2019. 
[25] T. Watanabe, Y. Kimura, and T. Ono, "Microfluidic fabrication of monodisperse polylactide microcapsules with tunable structures through rapid precipitation," Langmuir, vol. 29, no. 46, pp. 14082-14088, 2013.

[26] T. Govender, S. Stolnik, M. C. Garnett, L. Illum, and S. S. Davis, "PLGA nanoparticles prepared by nanoprecipitation: drug loading and release studies of a water soluble drug," Journal of Controlled Release, vol. 57, no. 2, pp. 171-185, 1999.

[27] X. Yu, Z. Zhao, W. Nie et al., "Biodegradable polymer microcapsules fabrication through a template-free approach," Langmuir, vol. 27, no. 16, pp. 10265-10273, 2011.

[28] S. R. Abulateefeh and A. M. Alkilany, "Synthesis and characterization of PLGA shell microcapsules containing aqueous cores prepared by internal phase separation," AAPS PharmSciTech, vol. 17, no. 4, pp. 891-897, 2016.

[29] P. Blasi, S. Giovagnoli, A. Schoubben et al., "Preparation and in vitro and in vivo characterization of composite microcapsules for cell encapsulation," International Journal of Pharmaceutics, vol. 324, no. 1, pp. 27-36, 2006.

[30] R. L. McCall and R. W. Sirianni, "PLGA nanoparticles formed by single- or double-emulsion with vitamin E-TPGS," JoveJournal of Visualized Experiments, vol. 82, Article ID 51015, 2013.

[31] A. Sahin, F. Spiroux, I. Guedon et al., "Using PVA and TPGS as combined emulsifier in nanoprecipitation method improves characteristics and anticancer activity of ibuprofen loaded PLGA nanoparticles," Die Pharmazie, vol. 72, no. 9, pp. 525-528, 2017.

[32] S. K. Paswan and T. R. Saini, "Purification of drug loaded PLGA nanoparticles prepared by emulsification solvent evaporation using stirred cell ultrafiltration technique," Pharmaceutical Research, vol. 34, no. 12, pp. 2779-2786, 2017.

[33] A. S. Ahmed, U. K. Mandal, M. Taher, D. Susanti, and J. M. Jaffri, "PVA-PEG physically cross-linked hydrogel film as a wound dressing: experimental design and optimization," Pharmaceutical Development and Technology, vol. 23, no. 8, pp. 751-760, 2018.

[34] N. Y. Martinez, P. F. Andrade, N. Durán, and S. Cavalitto, "Development of double emulsion nanoparticles for the encapsulation of bovine serum albumin," Colloids and Surfaces B: Biointerfaces, vol. 158, pp. 190-196, 2017.

[35] K. Shi, F. Cui, H. Yamamoto, and Y. Kawashima, "Optimized preparation of insulin-lauryl sulfate complex loaded poly (lactide-co-glycolide) nanoparticles using response surface methodology," Die Pharmazie, vol. 63, no. 10, pp. 721-725, 2008.

[36] R. Tanaka, T. Hayashi, M. Ike, Y. Noto, and T. K. Goto, "Reduction of dark-band-like metal artifacts caused by dental implant bodies using hypothetical monoenergetic imaging after dual-energy computed tomography," Oral Surgery, Oral Medicine, Oral Pathology and Oral Radiology, vol. 115, no. 6, pp. 833-838, 2013.

[37] A. C. Oenning, R. Jacobs, R. Jacobs et al., "Cone-beam CT in paediatric dentistry: DIMITRA project position statement," Pediatric Radiology, vol. 48, no. 3, pp. 308-316, 2018.

[38] G. Li, S. Luo, C. You et al., "A novel calibration method incorporating nonlinear optimization and ball-bearing markers for cone-beam CT with a parameterized trajectory," Medical Physics, vol. 46, no. 1, pp. 152-164, 2019. 